\title{
Treatment Recommendation System
}

\author{
Phong Thanh Nguyen, M. Ilayaraja, K. Shankar, Wahidah Hashim, Andino Maseleno
}

\begin{abstract}
In health care service the treatment recommendation system is plays very important role. In health care services for decision making the Treatment Recommender Systems (TRS) presented as a complementary tools. In current process the Treatment Recommender Systems increase the technology usability and reduce overloading of information.
\end{abstract}

Keywords : health sevices; Treatment Recommender ; complementary tools.

\section{INTRODUCTION}

At the point when the recommender system utilized as a piece of health industry then it is known as treatment Recommender Systems. This kind of recommender system can use by the client as close to home wellbeing exhorting instruments or by the doctors as a symptomatic help [1]. For getting to the data and suggestion of wellbeing the web is turned into the primary hotspot for client. The recommender system predicts the inclination or constancy of a client for a substance or thing. It can consider as a class of data sifting framework. This recommender system essentially utilized for suggesting the news, articles, recordings, books and pages on web. In the field of social insurance for customized care of patient the suggestion framework incorporate basic leadership process, among restorative specialists it can use for recognize the key feeling pioneers, in arranging the customized treatment the proposal framework bolster the patients to discover protection human services, the proposal framework give direction identified with customized medicinal services, and the procedure of prescribe a patient to a specialist dependent on the historical backdrop of past meeting [2].
Revised Manuscript Received on July 22, 2019.

* Correspondence Author

Phong Thanh Nguyen*, Department of Project Management, Ho Chi Minh City Open University, Vietnam. E-mail: phong.nt@ou.edu.vn

M. Ilayaraja, Department of Computer Science and Information Technology, Kalasalingam Academy of Research and Education, Krishnankoil, India. E-mail: ilayaraja.m@klu.ac.in

K. Shankar*, Department of Computer Applications, Alagappa University, Karaikudi, India. E-mail: shankarcrypto@gmail.com

Wahidah Hashim, Institute of Informatics and Computing Energy, Universiti Tenaga Nasional, Malaysia.

Andino Maseleno, Institute of Informatics and Computing Energy, Universiti Tenaga Nasional, Malaysia.

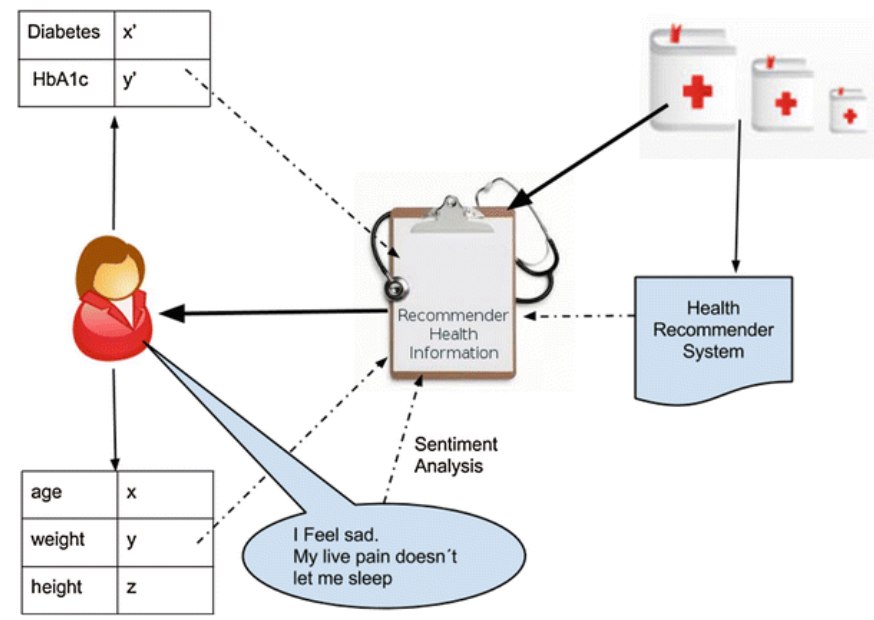

Figure 1: Example of treatment recommendation system

\section{LITERATUE SURVEY}

The first paper on Recommender systems was published in mid 1990's. From that time the Recommender systems field become the important research area. There are many deficiencies in the research classifications and literature review although the research on recommender systems has significantly increased in last 10 years.

Tapestry was use as a manual CF mail system [18], it was the oldest recommender systems. A collaborative filtering approach was applied by first recommender system that was based on computer and in mid nineties they are emerged [19, 20]. For finding news articles the GroupLens was known as $\mathrm{CF}$ recommendation system. The implementation with Mosaic browser interface of Bellcore video recommender algorithm was analyzed and evaluate by the author in [20]. To give personalized music recommendations Ringo used tast similarities. Based on item attributes, some prototypes like NewsWeederand InfoFinder by using CBF can provide news and documents [21, 22].

\section{RECOMMENDER SYSTEMS}

With respect to data mining and data cleaning the recommender systems become the important tool due to "big data" piling up on internet. To retrive the information accurately and effectively in earky 90's the information filtering techniques were used. Form a particular kind of information filtering (IF) technique this technique is known as recommender systems. This syetem provide the informations that are useful for user and present that information like books, news, webpages, movies, music, news etc. manily tree tyoes of filtering in Recommender Systems [2] are there: 
1. Collaborative Filtering Recommender Systems : this type of systems relies on the knowledge and informations that are composed and collected from users. AmazonTM is the example of such system.

2. Content-based Filtering Recommender Systems : this type of systems relies on the knowledge knowledge and informations that aggregated from unit descriptions of previous data and the users. Last.fmTM is the example of such system.

3. Hybrid Filtering Recommender Systems: this system is generally the combination of several techniques and approaches. Basically it is the combination of content based filtering and collaborative based filtering Recommender Systems.

\section{RECOMMENDER SYSTEM PHASES}

There are mainly three phases of recommender system are described as follows:

1. Phase of information collection

This phase is used to collect all the informations of patients and on the basis of attribute of patients, and patient's resources or behaviors it craete a patient profile. A recommender sytem cannot behave efficienly if it will not develop a well defined profile of a patinet. There are several inputs used in this phase like explicit, implicit and hybrid feedback. The recommender system is based on these inputs and these inputs are collected from differet ways. On an item the explicit feedback take input that is based on the intrest of patients. Through observing the behavior of a patient the implicit feedback takes indirectly preferences of patients [23].

\section{Phase of learning}

The assessment that are collected from previous phases are consider as input in this phase. To exploit the features of patient as output using learning algorithm to processes this feedback $[23,24,25]$.

3. Phase of Prediction or Recommender

In this pahse the items that are preferable are recommended. In inforamtion collection phase the feedback is analyzed, through the model a prediction can craete and it can analyze the obserbed activiltis and memory based activities of the patients $[23,24]$. recommender system phases are shown in figure below:

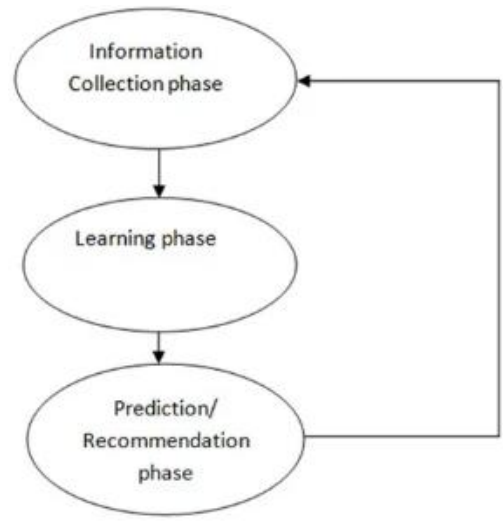

Figure 2: recommender system phases

\section{FILTERING BASED RECOMMENDER SYSTEM TYPES}

On the basis of availability of recommender system there are mainly three type of filtering based recommender system are shown in figure geuven below:

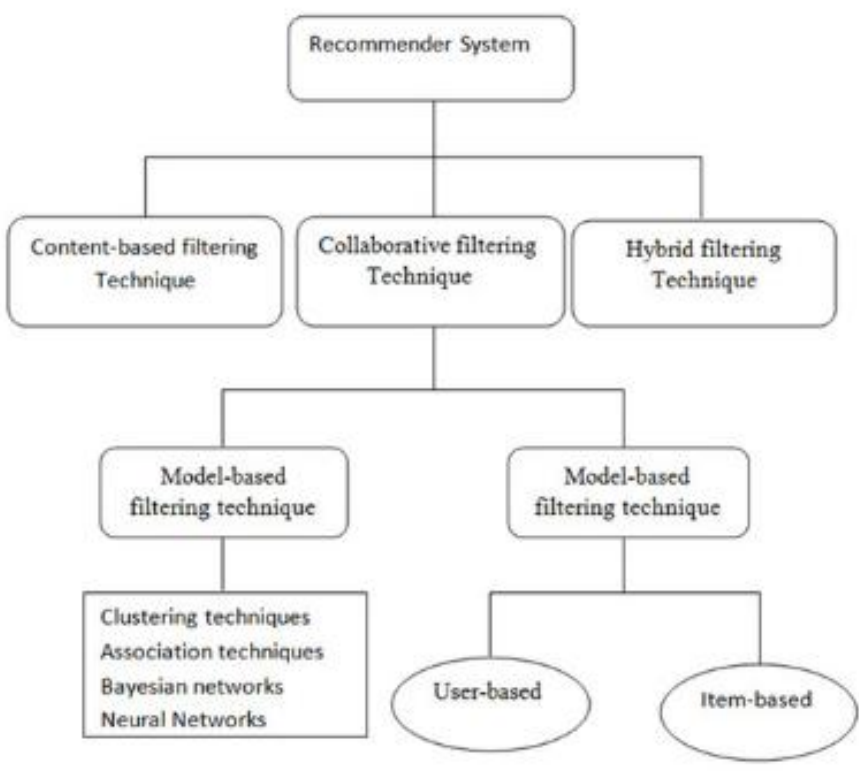

Figure 3: Type Recommender System based on filtering.

\section{HEALTH RECOMMENDER SYSTEMS BASED ON HEALTH}

When the recommender system used as a part of health industry then it is known as Health Recommender Systems. This type of recommender system can use by the user as personal health advising tools or by the physicians as a diagnostic assistance [2]. For accessing the information and recommendation of health the internet is become the main source for user. Fernandez et al defined that on the internet the health information can searched as follows [3]:

1. Tutorials, web blogs, images, forums, videos etc.

2. Multimedia resources like information about herbal cures of cancer, resources on autopsies etc.

3. The research papers that presented by medical patients, governments, organizations etc. 


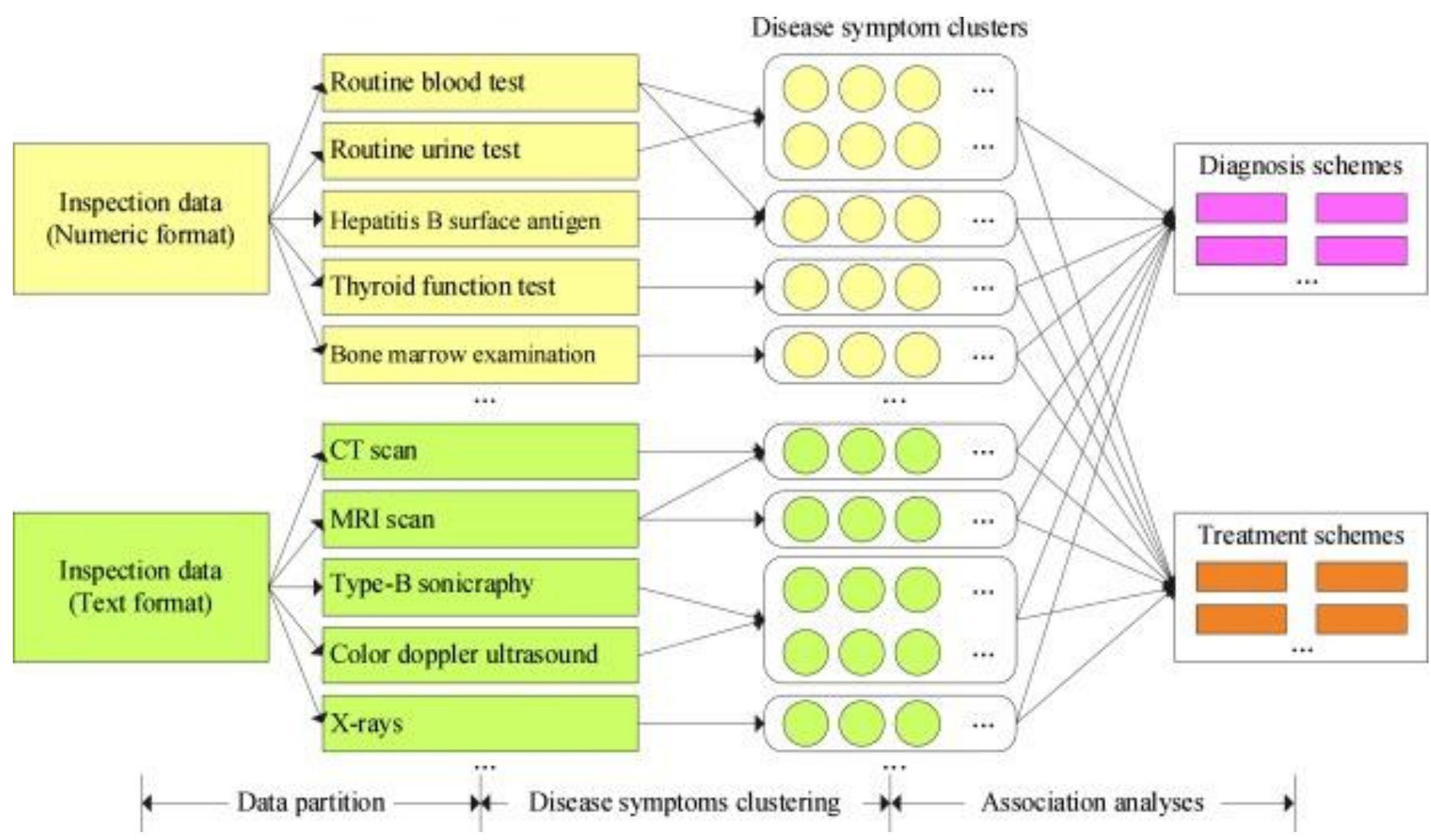

Figure 4: an example of diagnosis of disease and treatment recommendation system

\section{RECOMMENDER SYSTEMS IN HEALTHCARE}

The recommender system predicts the preference or fidelity of a user for an entity or item. It can consider as a class of information filtering system. This recommender system basically used for recommending the news, articles, videos, books and web pages on internet [4]. In the field of healthcare for personalized care of patient the recommendation system include decision-making process [5], among medical practitioners it can use for identify the key opinion leaders [6], in planning the personalized therapy the recommendation system support the patients to find preventative healthcare [7], the recommendation system give guidance related to personalized healthcare [8], and the process of recommend a patient to a doctor based on the history of previous consultation [9].

\section{RECOMMENDER SYSTEM USED FOR PERSONALIZED TREATMENT}

To understand and explore the relationship between covariates of patient and to understand the effectiveness of different options of treatment the Medical practitioners use survival models. These covariates include genetic and clinical features. to model treatment interaction at an individual level the Standard survival models like the linear Cox proportional hazards model need prior medical knowledge or extensive feature engineering. The nonlinear survival methods like survival forests and neural networks can model inherently these terms.

\section{Methodology}

For survival analysis the researchers used three main types of neural networks. These neural networks include the Variants of:

1. models of classifications

2. methods that are time encoded

3. methods based on prediction of risk

The risk prediction methods is a kind feed-forward neural network (NN) that calculate the risk of failure.

In Cox proportional hazards model, the network of Faraggi-Simon is a nonlinear extension.

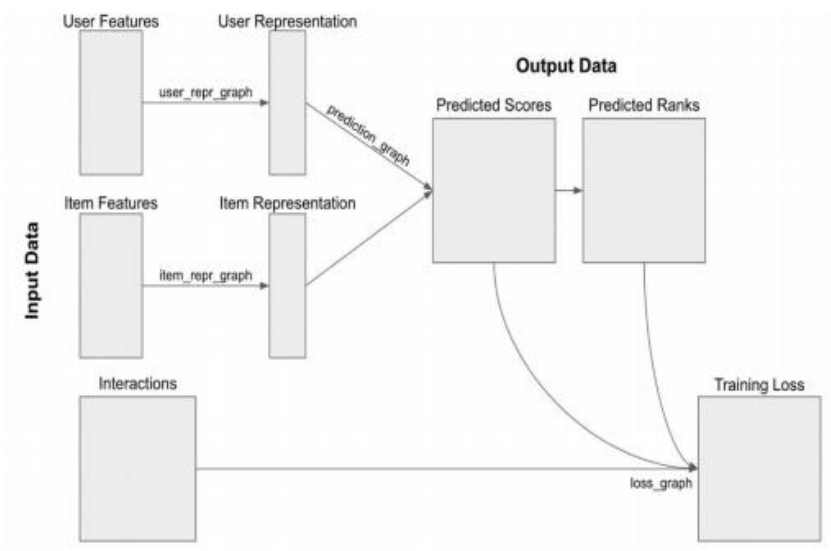

Figure 5: Deep learning Recommendation system 


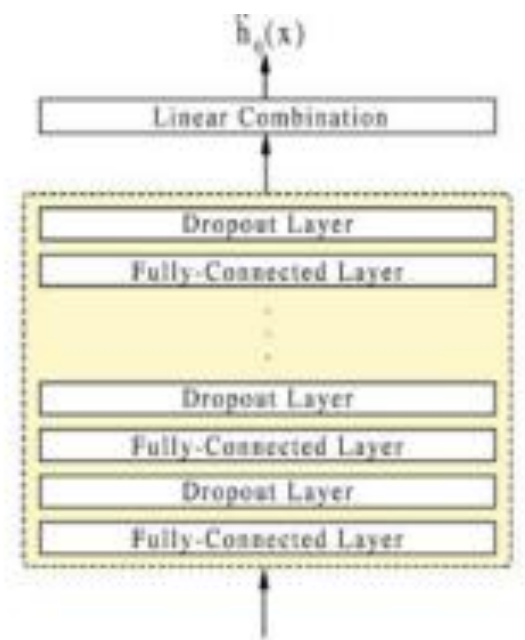

Figure 6: DeepSurv diagram

\section{FOR PERSONAL WELL-BEING SERVICES A SMART RECOMMENDER BASED ON HYBRID LEARNING}

The process of calculating the term of satisfaction with the positive outcomes can be considered as well being. The World Happiness Report shows that for personal being on the personal and social level the health and economy is very critical issue [10, 11]. With physical activities, nutrition, healthy lifestyle and personal behavior the diseases can prevent. By doing improvements in energy level, good healthy feelings and life expectancy the cost of treatment can reduce [12-15].

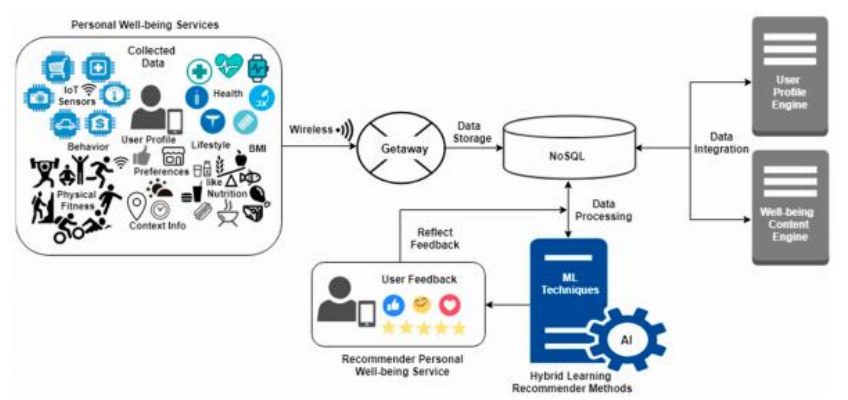

Figure 7: well being recommender services

In a specific field if user need particular information the task of the recommender system (RS) is to provides provide correct information. This approach considers recommender system as successful software tools [16]. For providing the recommendations of users there are many successful recommender system are used the example of such systems are Netflix, YouTube, Amazon and many more. The objective of these systems is to provide personalized contents to user [17].

\section{CONCLUSION}

As for information mining and information cleaning the recommender systems becomes the significant instrument because of "huge information" heaping up on web. To retrieve the data precisely and successfully in early 90's the data sifting strategies were utilized. Structure a specific sort of data separating strategy this method is known as recommender systems. This system give the information that are helpful for client and present that data like books, news, website pages, motion pictures, music, news etc. in social insurance administration the treatment suggestion framework is assumes significant job. In social insurance administrations for basic leadership the Treatment Recommender Systems (TRS) displayed as an integral apparatuses. In current procedure the Treatment Recommender Systems increment the innovation convenience and lessen over-burdening of data.

\section{ACKNOWLEDGMENT}

This article has been written with financial support of RUSA-Phase 2.0 grant sanctioned vide Letter No. F. 24-51/2014-U, Policy (TNMulti-Gen), Dept. of Edn. Govt. of India, Dt. 09.10.2018.

\section{REFERENCES}

1. Sezgin, Emre \& Ozkan, Sevgi. (2013). A systematic literature review on Health Recommender Systems. 2013 E-Health and Bioengineering Conference, EHB 2013. 1-4. 10.1109/EHB.2013.6707249.

2. F. Ricci, L. Rokach, B. Shapira and P. B. Kantor, Introduction to Recommender Systems Handbook, pp. 257-297, Springer, Berlin, 2011

3. L. Fernandez-luque, R. Karlsen and L.K. Vognild, "Challenges and Opportunities of using Recommender Systems for Personalized Health Education" Stud. Health Technol. Inform., 150(903), pp. 903-7, 2009.

4. J. B. Schafer, J. Konstan, and J. Riedl, "Recommender systems in ecommerce," in Proceedings of the 1st ACM Conference on Electronic Commerce (EC), 1999, pp. 158-166.

5. E. Sezgin and S. Ozkan, "A systematic literature review on health recommender systems," in E-Health and Bioengineering Conference (EHB), 2013. IEEE, 2013, pp. 1-4.

6. L. Guo, B. Jin, C. Yao, H. Yang, D. Huang, and F. Wang, "Which doctor to trust: A recommender system for identifying the right doctors." Journal of Medical Internet Research, vol. 18, 2016.

7. F. Graber, S. Beckert, D. Kuster, J. Schmitt, S. Abraham, H. Malberg, and S. Zaunseder., "Neighborhood-based collaborative filtering for therapy decision support," Journal of Healthcare Engineering, 082017.

8. H. Schafer, S. Hors-Fraile, R. P. Karumur, A. Calero Valdez, A. Said, ". H. Torkamaan, T. Ulmer, and C. Trattner, "Towards health (aware) recommender systems," in Proceedings of the 2017 International Conference on Digital Health (DH). ACM, 2017, pp. 157-161.

9. Q. Han, I. Mart'inez de Rituerto de Troya, M. Ji, M. Gaur, and L. Zejnilovic, "A collaborative filtering recommender system in primary care: Towards a trusting patient-doctor relationship," in 2018 IEEE International Conference on Healthcare Informatics (ICHI), June 2018, pp. 377-379.

10. Prendergast, K.B.; Schofield, G.M.; Mackay, L.M. Associations between lifestyle behaviours and optimal wellbeing in a diverse sample of New Zealand adults. BMC Public Health 2016, 16, 62. [CrossRef] [PubMed].

11. Helliwell, J.; Layard, R.; Sachs, J. World Happiness Report; Sustainable Development Solutions Network: New York, NY, USA, 2018; ISBN 978-0-9968513-6-7.

12. National Center for Chronic Disease Prevention and Health Promotion Home Page. Available online: https://www.cdc.gov/chronicdisease (accessed on 20 September 2018).

13. Sodhro, A.H.; Kumar, Z.L.; Baik, S.W. Mobile edge computing based QoS optimization in medical healthcare applications. Int. J. Inf. Manag. 2018. [CrossRef]

14. Singh, D.; Tiwary, U.S.; Chung, W.Y. IP-based Ubiquitous Healthcare system. In Proceedings of the International Conference on Control, Automation and Systems (ICCAS 2008), Seoul, Korea, 14-17 October 2008; pp. 131-136.

15. Singh, D.; Tiwary, U.S.; Lee, H.J.; Chung, W.Y. Global Healthcare Monitoring System using 6lowpan Networks. In Proceedings of the 11th International Conference on Advanced Communication Technology (ICACT-2009), Phoenix Park, Korea, 15-18 February 2009; pp $113-117$. 
16. Adomavicius, G.; Tuzhilin, A. Toward the next generation of recommender systems: A survey of the state-of-the-art and possible extensions. IEEE Trans. Knowl. Data Eng. 2005, 17, 734-749.

17. Amatriain, X. Mining Large Streams of User Data for Personalized Recommendations. ACM SIGKDD Explor. Newsl. 2013, 14, 37-48.

18. D. Goldberg, D. Nichols, B. M. Oki, D. Terry, Using collaborative filtering to weave an information tapestry,Commun. ACM 35 (12) (1992) 61-70.

19. P. Resnick, N. Iacovou, M. Suchak, P. Bergstrom, J. Riedl, Grouplens: An open architecture for collaborativefiltering of netnews, in: Proceedings of the 1994 ACM Conference on Computer Supported Cooperative Work,CSCW '94, ACM, New York, NY, USA, 1994, pp. 175-186. doi:10.1145/192844.192905. 